\title{
Gestión integral de activos fijos con enfoque en el control contable administrativo
}

\author{
Integral management of fixed assets with focus on administrative accounting \\ control
}

Lady Alexandra García Jiménez. ${ }^{1}$, Cecilia Ivonne Narváez Zurita. ${ }^{2}$ \& Juan Carlos Erazo Álvarez. ${ }^{3}$

DOI: https://doi.org/10.33262/visionariodigital.v3i2.479

\begin{abstract}
.
Nowadays, the Fixed Asset Management Models have become important tools for the easy achievement of strategic objectives within organizations. Therefore analyze their behavior, reduce costs, and maximize their investments are processes of great significance at the moment when a value is produced.

This article submits the proposal of a Fixed Asset Integral Management Model based on three main components: planning, execution, control, and performance. The particular feature of this model is the organization of the accounting system and administrative to leverage the resources of the organization and reach its maximum performance responsibly and sustainably.

To achieve the development of the model first a phase of Literature analysis related to the topic was carried out. Next, a diagnostic was made in the Agroartesenal Association of Ecological Producers of Palanda and Chinchipe from the province of Zamora Chinchipe, Ecuador. Then, a correlation matrix of the results was established, and subsequently, the model structure was sketched. Finally, each of its components was described.
\end{abstract}

Keywords: Fixed Assets, Integral Management, Audit Control, Administrative Control, Depreciation, Revaluation.

\footnotetext{
${ }^{1}$ Universidad Católica de Cuenca, Departamento de Posgrado, Cuenca, Ecuador, ladyalexandragarciajimenez@gmail.com

${ }^{2}$ Universidad Católica de Cuenca, Departamento de Posgrado, Cuenca, Ecuador, inarvaez@ucacue.edu.ec

${ }^{3}$ Universidad Católica de Cuenca, Departamento de Posgrado, Cuenca, Ecuador, jcerazo@ucacue.edu.ec
} 


\section{Resumen.}

En la actualidad los modelos de gestión de activos fijos se han convertido en herramientas que facilitan la consecución de los objetivos estratégicos en las organizaciones; por tanto, analizar su comportamiento, reducir sus costos y optimizar sus inversiones son procesos que revisten de gran importancia al momento de producir valor.

En este artículo se presenta la propuesta de un modelo de gestión integral de activos fijos, sustentado en cuatro ejes: planificación, ejecución, control y actuación. El modelo tiene como característica particular la articulación de los procesos contables y administrativos en forma práctica, de manera tal, que se aprovecha los recursos de la organización para alcanzar su máximo rendimiento de manera responsable y sostenible.

Para el desarrollo del modelo se inició con una fase de análisis de la literatura referente al tema, posteriormente se realizó un diagnóstico en la Asociación Agroartesanal de Productores Ecológicos de Palanda y Chinchipe de la provincia de Zamora Chinchipe, Ecuador, luego se estableció una matriz de correlación de resultados, se bosquejó la estructura del modelo y finalmente se describió cada uno de sus componentes.

Palabras claves: Activo Fijo, Gestión Integral, Control Contable, Control Administrativo, Depreciación, Revalorización.

\section{Introducción.}

Santos (2008) y Agudelo (2013) expresan que en la actualidad un gran número de organizaciones, consideran a los activos fijos como un elemento determinante en sus planes estratégicos para alcanzar los objetivos planteados y sobre todo el éxito, por ende resulta imprescindible establecer una dinámica específica relacionada con su administración y mantenimiento, de tal manera que contribuyan a la generación de valor en la entidad, asegurando su estabilidad, competitividad y crecimiento en el mercado, obtenida a través del aprovechamiento correcto de la capacidad máxima de los activos en los procesos operativos y productivos, la reducción de sus costos siempre y cuando esto no conlleve a repercusiones negativas en su desempeño, disminuir su incidencia en el ambiente y minimizar los riesgos.

Una gestión integral de activos debe involucrar el área operativa, proyectos e ingeniería, área administrativa, productiva, adquisiciones y mantenimiento, esto con el fin de coadyuvar al mejoramiento de la confiabilidad de los activos y favorecerse de los resultados, a través de la aplicación de un cúmulo de procesos y herramientas que permiten hacer frente a la vertiginosa competencia y a las variaciones en el ambiente económico, incluyendo estrategias para disminuir e incluso evitar el mal uso y desperdicio de los recursos. 
Normas Internacionales de Contabilidad (NIC).

Monge (2005) y Espejo (2007) manifiestan que desde la perspectiva empresarial existe un amplio escenario de la realidad comercial, tecnológica y social, así como la alternativa de comerciar en cualquier mercado financiero de los diferentes países, por ende, es necesario establecer normas que regulen el actuar en el entorno contable, pudiendo ser asimilados por los usuarios de la información económica - financiera de las organizaciones y así posibilitar el contraste de los datos y aumentar la transparencia de los mercados.

Por tanto, las NIC constituyen un conjunto de normas contables de interés público, elaboradas y emitidas por el International Accounting Standards Committee (de aquí en adelante IASC), que regulan la presentación de los estados financieros; gozando de aceptación en los diferentes países, siendo observadas y aplicadas rigurosamente por empresas de todo el mundo. Debe señalarse que las normas internacionales consideran el análisis de los activos fijos directamente en la Norma Internacional de Contabilidad (NIC) 16, siendo necesario primero indicar que los activos fijos también se denominan propiedad, planta y equipo.

Coral (2013) afirma que la NIC 16 tiene como finalidad señalar la contabilización de dichos activos, el establecimiento de su importe en libros, cargos por concepto de depreciación y pérdidas ocasionadas por el deterioro, que constituyen el principal problema que se identifica en el reconocimiento contable; de tal manera que los interesados en los estados financieros dispongan de suficiente información relativa a los activos para su uso o producción, ayudando en la obtención de rentabilidad en un periodo de tiempo determinado en concordancia con su enfoque social.

La NIC 16 toma en consideración diferentes componentes en lo que corresponde al registro contable de los activos fijos, entre ellos se resaltan los siguientes:

a. Medición en el momento del reconocimiento.

Para que un activo fijo sea reconocido como tal, debe existir la garantía que en el futuro se obtendrá algún provecho económico procedente del mismo, la medición de su costo debe ser fidedigno y será útil a la entidad por un largo periodo de tiempo.

Siendo las piezas de repuesto y el equipo auxiliar normalmente considerado en calidad de inventarios, se registrará su consumo dentro de los resultados al finalizar el periodo. Por otra parte, las piezas de repuesto significativas y el equipo de mantenimiento que se tenga de forma permanente, estimados a ser empleados en más de un periodo, reúnen generalmente las cualidades para ser reconocidos como elementos de propiedades, planta y equipo.

De igual forma, la búsqueda permanente para incrementar la seguridad y la preocupación por el aspecto medioambiental constituyen los principales motivos para adquirir elementos de la 
partida de propiedades, planta y equipo. Si bien dicha adquisición no conlleva a un aumento de los beneficios económicos que se derivan de los elementos de la partida en mención existentes, coadyuvan a que adicionalmente la empresa se beneficie de forma económica de los activos restantes. Los costos de fuerza de trabajo y consumibles que se deban efectuar para el mantenimiento diario del elemento, conocidos con la denominación de reparaciones y conservación del elemento de propiedades, planta y equipo, deberán ser reconocidos en el resultado más no en el valor en libros de un elemento de propiedades, planta y equipo.

Un elemento de propiedades, planta y equipo, que reúna las características para ser considerado como un activo, debe medirse por su costo, el cual comprende: el valor cancelado al momento de su adquisición, considerando los aranceles por la importación e impuestos indirectos que no sea posible recuperarlos, una vez deducido cualquier rebaja del importe a pagar, y adicionalmente, tomando en cuenta los desembolsos monetarios que se efectúen para ubicar el activo en un lugar adecuado de tal manera que esté en capacidad para operar de la forma prevista.

b. Medición posterior al reconocimiento.

Una vez reconocido los activos como tales, la entidad goza de libertad para elegir el modelo de medición posterior al reconocimiento más conveniente acorde a sus características y posteriormente legitimarlo para su aplicación como parte de sus políticas contables. Cabe indicar que si el reconocimiento del costo de un elemento se efectúa luego de deducir del mismo, el monto de la depreciación acumulada y el total de las pérdidas generadas como consecuencia del deterioro, la entidad estaría empleando el modelo del costo. No obstante, si emplea el valor razonable y disminuye su depreciación acumulada y las pérdidas totales ocasionadas por el deterioro de valor, estaría utilizando el modelo de revaluación.

Con la finalidad de mantener la concordancia y reducir la variación entre el importe en libros y el valor razonable determinado, resulta imprescindible efectuar una revaluación con regularidad. Como producto de la revaluación se puede obtener un aumento o una disminución del importe en libros: en el primer caso se deberá identificar en el resultado integral y se reflejará en el patrimonio empleando la denominación de superávit de revaluación, en el segundo caso deberá ser identificado y registrado en el resultado del ejercicio. No obstante, si se cuenta con saldo acreedor en el superávit de revaluación del activo, la disminución podrá reconocerse en otro resultado integral (IASB, 2005).

Bernal (2004); Espejo (2007); Parkin, Esquivel y Muñoz (2007) y Coral (2013) coinciden que las propiedades, planta y equipo a excepción de los terrenos pierden su eficiencia, efectividad y capacidad de servicio, debido al desgaste natural por el uso continuo o porque pueden volverse obsoletos a causa de los avances tecnológicos, extinguiéndose su período de vida útil y perdiendo progresivamente su valor con el tiempo; por ende es preciso recurrir a la depreciación para reconocer tal hecho. 
Es por ello que, al finalizar la vida útil de un activo, éste conservará un valor residual, pudiendo ser objeto de enajenamiento, si se descuenta dicho valor de su costo inicial se obtiene su importe depreciable, el mismo que en forma periódica, proporcional y razonable, debe ser imputado a cada periodo por el lapso de vida útil del activo, siendo este proceso denominado depreciación. Para ello el IASB (2005) señala que será imperioso efectuar el cálculo de la depreciación en forma aislada para cada parte que integre el elemento de propiedades, planta y equipo cuando su costo sea relevante en relación al costo global del elemento. Cabe indicar que un activo empezará a depreciarse desde el momento que se encuentre en el lugar y condiciones favorables para su utilización y cesará cuando el activo se catalogue como mantenido para enajenarse o al momento en que se realice la baja en cuentas del mismo, lo que ocurra primero, así mismo un activo seguirá depreciándose incluso cuando no esté prestando sus servicios momentáneamente al ente económico o se haya separado de la utilización activa, salvo que esté depreciado en su totalidad.

Para tal efecto, existen varios métodos de depreciación, entre ellos se puede citar: legal, línea recta, alícuotas unidades de producción y acelerado o suma de dígitos, de los cuales la entidad analizará y empleará el más conveniente, teniendo presente la forma cómo se consumirá los beneficios económicos que genere el activo en el futuro. En el Ecuador, la depreciación se efectúa tomando en consideración lo establecido por la administración tributaria (Servicio de Rentas Internas - SRI), la misma que establece que los porcentajes de depreciación de los activos fijos estarán de conformidad a lo que estipula el Art. 28 del Reglamento a la Ley Orgánica de Régimen Tributario Interno (LORTI), tal como se muestra en la tabla 1:

Tabla 1 Años de vida útil y porcentajes de depreciación

\begin{tabular}{lcc}
\hline \multicolumn{1}{c}{ Activo fijo } & $\begin{array}{c}\text { Porcentaje de } \\
\text { depreciación anual }\end{array}$ & $\begin{array}{c}\text { Años de vida } \\
\text { útil }\end{array}$ \\
\hline Inmuebles (excepto terrenos). & $5 \%$ & 20 años \\
Instalaciones, maquinarias, equipos y & $10 \%$ & 10 años \\
muebles. & & \\
Vehículos, equipos de transporte. & $20 \%$ & 5 años \\
Equipos de cómputo y software. & $33 \%$ & 3 años \\
\hline
\end{tabular}

Adaptado de: (Espejo, 2007; SRI, 2015)

\section{c. Baja en cuentas.}

Para este propósito existen algunos motivos por los cuales el valor en libros de un elemento de propiedades, planta y equipo podrá darse de baja, entre ellos: por su disposición que puede ser a través de la venta, en caso que no se prevea o sea poco probable beneficiarse económicamente por su utilización o disposición a futuro, hurto del activo fijo, destrucción por catástrofe fortuita, ciclo de depreciación cumplido y por obsolescencia. 
En este sentido, se podrán deducir las pérdidas producto del deterioro, destrucción, extravío y otros acontecimientos que incidan económicamente a las propiedades, planta y equipo utilizados en las operaciones que producen la renta y que sean por imprevistos, fuerza mayor o actos delincuenciales, solamente en la proporción en que esté desprotegida por indemnización o seguro y debiendo justificar tal pérdida mediante una declaración juramentada efectuada ante un notario o juez (Espejo, 2007).

Según el IASB (2005), el resultado que se obtenga como consecuencia de dar de baja en cuentas un bien, sea pérdida o ganancia, no se considerará como ingreso de operaciones ordinarias, pero si formarán parte del resultado del ejercicio. La variación que exista entre el valor neto obtenido mediante la disposición del elemento y el valor que se encuentra registrado en libros, permitirá establecer la pérdida o ganancia de la baja en cuentas.

Es así que, en base a los fundamentos abordados anteriormente, Candelario (2013) y Cerón, Orduña, Aponte y Romero (2015) consideran que con el fin de lograr un manejo óptimo y eficaz de los activos fijos de una entidad se debe adoptar a la gestión integral como el instrumento clave para garantizar este fin, misma que coadyuva a la planificación de actividades metódicas y tácticas, prácticas eficientes y políticas necesarias para un plan estratégico organizacional que contribuya al incremento del rendimiento, control del nivel de riesgo y gasto a lo largo de la vida útil de los activos fijos, a corregir falencias y a la generación de valor a través de su empleo.

\section{Beneficios de la gestión de activos fijos.}

Bedoya (2014) expresa que una adecuada gestión de activos ofrece una serie de beneficios a la entidad, entre los cuales se pueden destacar que: garantiza que las acciones ejecutadas sean acertadas y estén produciendo el beneficio esperado, usando el cien por ciento de la capacidad instalada de sus activos fijos; permite llevar un control riguroso de la ejecución de los planes para el mantenimiento y conservación de los activos; logra la disminución de fallas en el producto final; contribuye a la consecución y obtención de valor en tanto que equilibra los costos de índole financiero, del ambiente y la sociedad, mejora la productividad, la calidad del servicio y desenvolvimiento de los activos; garantiza la recuperación del dinero invertido en activos; controla durante la vida útil de los activos, los riesgos a los que se encuentran expuestos; prolonga la vida útil de los bienes.

Pilares de la gestión de activos fijos.

Amendola (como se citó en González , González y Esparza, 2017) y Amendola (2011) expresa que los pilares fundamentales de la gestión de activos son los siguientes: 
Figura 1. Pilares de la gestión de activos.

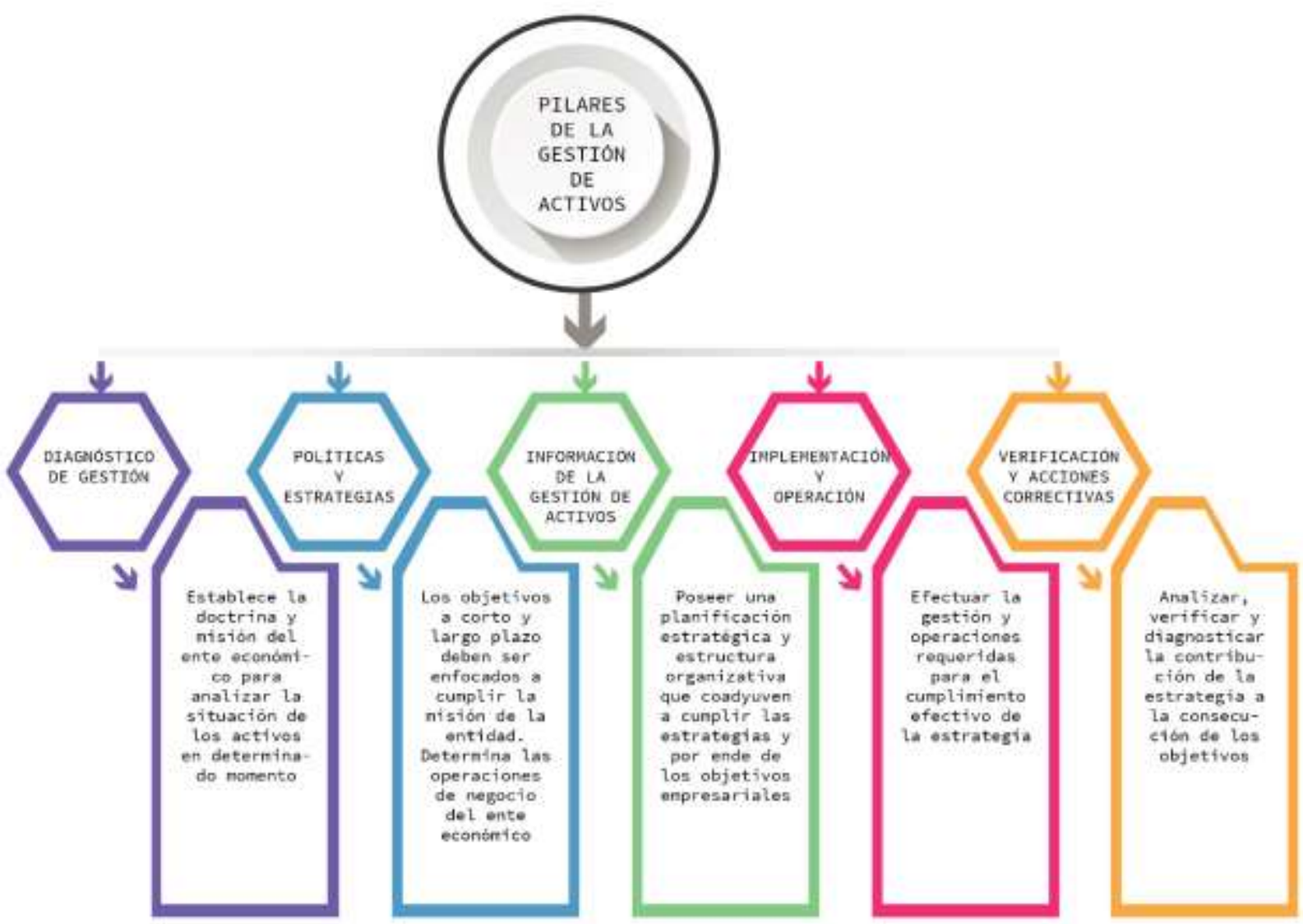

Modelos de gestión integral de activos fijos y su implementación.

En la literatura especializada se identifican principalmente 3 modelos de gestión de activos fijos, de los cuales Amendola (2011) señala dos:

a. Modelo de gestión asociado al sistema de gestión integral de activos físicos.- en este modelo intervienen: una fase de análisis y valoración, otra de actividades y conservación, de índices técnicos - financieros y finalmente de estrategias o técnicas de confiabilidad. Todo dentro de un marco de explotación de competencias certificadas, fortalecido con un aprendizaje íntegro y especializado. Adicionalmente los elementos del modelo deben receptar, procesar y generar información a través de su interrelación; así como también cada elemento simultáneamente ejecuta procedimientos internos relevantes.

b. Modelo conceptual GFMAM "Global Forum for Maintenance and Asset Management".- garantiza el potencial de la gestión de activos, concibiéndola como una fusión de principios, calidad en la realización de las transacciones y actitud comprometida de las personas con la institución; además brinda especial relevancia al discernimiento y 
habilidad para gestionar adecuadamente los activos. Su filosofía radica en los principios de la gestión de activos: perspectiva de salida, la capacidad, protección y enseñanza.

$\mathrm{Al}$ respecto, el autor plantea las siguientes 7 fases para implementar una adecuada gestión de activos en una entidad:

Fase 1: Diagnóstico y análisis de la situación actual: la alternativa más viable consiste en investigar y analizar las tendencias en gestión de activos físicos y contrastarlas con patrones empíricos reconocidos a nivel mundial como óptimos.

Fase 2: Análisis de criticidad: determinar qué aspectos serán objeto de análisis, considerando su enfoque y fundamentando con documentación. Los costos de mantenimiento, periodos de parada y eventualidades que disminuyen la producción, pueden ser parámetros de selección.

Fase 3: Estrategias de confiabilidad: consiste en la búsqueda de métodos y estrategias que coadyuven a la conservación de activos, a incrementar su eficiencia y disminuir los costos, conllevando a la formación de una programación equilibrada, que logre la interacción del mantenimiento: de corrección, prevención, predicción y proactivo.

Fase 4: Desarrollo del plan de mantenimiento de activos: dispondrá de tres tipos de operaciones: las acciones cotidianas a ser desempeñadas por el personal de mantenimiento, por los operarios de producción y actividades denominadas on/off tales como de reajuste o reparación, llevadas a cabo por especialistas de equipos.

Fase 5: Plan de acción de cobertura predictivo a implementar: establecer las estrategias a emplear y ejecutar y los elementos cuya intervención es necesaria para un control adecuado de los equipos, sean estos rotativos, permanentes, eléctricos e instrumentación.

Fase 6: Plan general de gestión integral de activos fijos: se enfocará en el mantenimiento proactivo, es decir localizará los inconvenientes en los equipos y maquinaria de la entidad y tomará las acciones necesarias para prevenir que ocurran nuevamente, así como conseguir que los activos incrementen su rendimiento.

Fase 7: Implementación de un modelo de confiabilidad con fundamento en la gestión integral de mantenimiento de activos: estimar los resultados tanto de gestión, así como monetarios del plan de gestión integral de activos fijos que involucrará las etapas de: planear, programar, dar seguimiento y vigilar el mantenimiento de los activos fijos, así como obtener datos para la elaboración de indicadores.

Cabe señalar que, la gestión integral puede ser considerada como una actividad enfocada a prevenir y detectar errores en los equipos de operaciones, no obstante, en varias ocasiones dicha labor no siempre resulta completamente táctica. En los últimos años los entes económicos, enfocados en potencializar su capacidad productiva y operar eficientemente, 
han automatizado gran parte de sus operaciones, dejando relegada la actividad manual. Por ende, la gestión integral procurará que las líneas de producción aprovechen al máximo sus estándares de diseño, garantizando una producción óptima.

Por otra parte, Agudelo (2013) propone un modelo de gestión de activos fijos con enfoque en procesos, que fusiona la gestión de calidad con la gestión de activos fijos de manera acertada y audaz para implementarla de forma sincrónica, con ello se atenua la posiblidad de duplicar la realización de tareas gastando esfuerzos innecesarios y que pueden aprovecharse de manera óptima; mejorando la disposición, accesibilidad, seguridad y confianza en la propiedad, planta y equipo, y emitiendo directrices que guían su comprensión y ejecución. El mencionado modelo involucra: el tratamiento y análisis de documentación y sus registros, elemento humano, abastecimiento de recursos, instalaciones, dispositivos para supervisar y medir, así como también una serie de labores que se pueden llevar a cabo para resarcir errores.

La búsqueda de un control íntegro de los activos fijos se debe abordar desde dos perspectivas: contable y administrativa. Respecto al control contable, Norka (2005); Carmenate (2013) y Contaduría General de la Nación (2015) manifiestan que este proceso lo constituyen los métodos, procedimientos, técnicas y planificación de la entidad orientados a la preservación de los activos y a garantizar que los informes y registros financieros sean veraces.

El control interno contable es de gran utilidad para verificar que se registren en su totalidad las operaciones que involucren activos fijos, en forma y momento oportuno, por la cuantía correcta, en las cuentas correspondientes y en el ejercicio contable en que fueron ejecutadas, con el propósito de coadyuvar a la preparación de los informes financieros; evitar que se presenten inconsistencias entre lo contabilizado y los activos físicos existentes, permitiendo efectuar una conciliación, y de la misma manera buscar el motivo que originó divergencias para tomar las medidas correctivas pertinentes; que en forma previa a la ejecución de cualquier labor que involucre los activos fijos se disponga del consentimiento general y específico para tal acción, de tal manera que se mantenga un control de su manejo (Estupiñán, 2015). También, resulta vital su utilización para procurar que las actividades de operación y custodia de activos fijos sean ejecutadas por personas completamente independientes del manejo de la contabilidad, esto con el fin de precautelar los intereses de la entidad y evitar condiciones favorables para que se ejecuten y oculten actos ilegales.

Según Mantilla y Cante (2005) para un adecuado control contable, en el mayor general se debe llevar una cuenta auxiliar por cada tipo de activo fijo, mismo que deberá incluir datos que permitan su fácil localización e identificación. La base de datos para un buen control contable de activos fijos, deberá contener: código de identificación física, código de equipo y modelo, descripción amplia del activo, fecha y documento de adquisición, costo y número de registro contable de la compra, área usuaria, depreciación anual y acumulada según lo establece la ley, valor neto del activo, estado actual y vida útil estimada. 
Por otra parte, en cuanto al control administrativo, Schermerhorn (2010); Ramírez (2013) y Estupiñán (2015) concuerdan que constituye un plan de organización que permite valorar el desenvolvimiento de los encargados respecto al uso de los recursos y activos fijos de la entidad, con el propósito de asegurar que sus acciones aporten al cumplimiento de la estrategia empresarial trazada, a satisfacer o superar los objetivos empresariales y contribuir a la generación de valor.

La importancia del control administrativo de activos fijos se ve influenciado por el vertiginoso y cada vez más agresivo ambiente en que se desenvuelven e interactúan las organizaciones, ocasionando que enfoquen sus esfuerzos en poseer un sistema de información capaz de verificar el nivel de eficiencia del empleo de recursos y activos fijos, así como estimar en qué nivel la empresa es capaz de generar valor para cada uno de sus componentes, alineados a los objetivos empresariales, considerando que la falta de compromiso en cuanto al control del sistema ocasiona deterioro y disminución de su valor. Cuando un ente económico actúa al margen de un sistema de control administrativo carece de información vital para identificar a tiempo las desviaciones o irregularidades y efectuar acciones correctivas con prontitud inherentes a su propiedad, planta y equipo. Por otra parte, el control administrativo también permite cuantificar los aciertos en la administración de activos fijos, con la finalidad que sean aprovechados en proyectos posteriores.

Cabe mencionar que, la responsabilidad del control administrativo de los activos fijos se delegará a un encargado, sin embargo, también corresponderá a los trabajadores. El responsable tendrá a su cargo: la recepción, adjudicación y registro de los muebles y enseres, equipos de cómputo y otros activos; la asignación de un código para su identificación; mantener, arreglar y sustituir repuestos de los activos fijos; constatar los bienes de manera regular, sin previo aviso y a solicitud de dependencias superiores; salvaguardar, fiscalizar y solicitar oportunamente el mantenimiento y arreglo de los activos custodiados.

Es importante mencionar que, se extinguirá la responsabilidad del funcionario respecto de los activos que se encontraban bajo su tutela, cuando se retornen los mismos al responsable de los bienes de la entidad, mediante un acta que respalde la operación. En cuanto al almacenamiento de los activos fijos devueltos, se designará un espacio específico para tal fin, que permita una adecuada salvaguarda de los bienes devueltos por el personal, ya sea por discontinuidad de vínculo laboral, variación en sus actividades, u obsolescencia de los bienes.

De la misma manera, existen ciertas medidas de seguridad que se deben observar y cumplir para el logro de una administración satisfactoria de los bienes, mismas que se detallan a continuación:

Equipos de computación: no estará permitida la manipulación de líquidos cerca de equipos de cómputo, deberá evitarse su exposición a la luz del sol en forma directa y factores 
medioambientales como polvo que pueda afectar su normal desempeño. El espacio donde se ubiquen las computadoras estará acondicionado con una fuente de energía eléctrica segura.

Equipos de fotocopiadora: se delegará a un trabajador responsable de la tutela del bien, quien conocerá acerca de su manejo correcto, con el fin de garantizar su preservación y solicitud del servicio de reparación en caso que sea necesario.

Vehículos: las obligaciones, horarios y restricciones referentes a la utilización, protección y custodia de los vehículos deberá ser regulada a través de un reglamento interno.

Mantenimiento de inmuebles, equipos y vehículos: la responsabilidad del mantenimiento preventivo recae sobre el encargado de activos fijos, quién dará fiel cumplimiento al cronograma definido, verificará el servicio de mantenimiento y reemplazo de repuestos, emitiendo regularmente un informe sobre el estado actual de los bienes; sin embargo, en caso de suscitarse alguna avería en éstos, el trabajador encargado de su custodia, deberá solicitar oportunamente el arreglo.

Instalaciones eléctricas: Los trabajadores deberán ser cuidadosos y colaborar apagando los equipos y desconectarlos una vez terminada la jornada laboral.

Equipos de incendio: revisar que los extintores estén funcionando correctamente, estén recargados, no presenten ninguna anomalía, que cuenten con la capacidad necesaria, que estén colocados estratégicamente como mínimo a $10 \mathrm{~cm}$. del suelo y $130 \mathrm{~cm}$. como máximo.

En el mismo sentido, tomando como base lo establecido por la Contraloría General del Estado (2016) en lo referente a la identificación de bienes de larga duración y acoplando estos preceptos al sector privado, se concluye que en forma permanente se deberá identificar y revisar los activos fijos de la entidad, así como preparar un registro que señale el estado real, conteo físico y conciliar con los registros de contabilidad.

Entre las principales técnicas de identificación de los activos fijos se encuentran:

Plaqueo: emplea una placa o etiqueta que funciona con un sistema de código de barras que da origen a una interfase con la base de información principal para la identificación de los elementos de propiedad, planta y equipo inventariados.

Marcación: consiste en identificar los bienes a través del marcado que se efectúa directamente en el sistema de empaque, ya sea imprimiendo dicha marca o empleando rótulos que se adhieran, etiquetas o algún tipo de sticker.

Etiquetado: las etiquetas se ubican sobre las placas, su principal beneficio es que contribuyen a la economía y es relativamente fácil reemplazarlas o enmendarlas, ya que la impresión de las etiquetas se puede efectuar al momento que se ingresa el bien al sistema. 
Código de barra: consiste en un grupo de líneas paralelas de diferente dimensión y espaciado, que constituyen cadenas de caracteres y de forma conjunta almacenan un código específico, permitiendo el reconocimiento instantáneo de un bien y sus características.

Lector de chip: las tarjetas RFID constituyen unos dispositivos de pequeña dimensión que se asemejan a una pegatina, y pueden ser adheridas a un activo fijo facilitando su identificación. Dispone de antenas que facilitan la recepción y respuesta a peticiones por radiofrecuencia.

En forma adicional es importante señalar, que las operaciones de la entidad deben estar orientadas a consolidar la estrategia empresarial, debido a que en ella se establecen las metas conjuntamente con las acciones que se requieren para alcanzarlas. En vista que es a través del control administrativo que se ejecutan las estrategias, las metas de la organización encierran una gran importancia por lo que podrían ser consideradas como la piedra angular para su diseño. Es así que, un sistema de control administrativo debe considerar el desarrollo en tres actividades principales, siendo éstas:

1. Establecer los objetivos y metas: los objetivos del sistema de control administrativo deben provenir de la estrategia organizacional, ser fijados en términos cuantificables y de fácil valoración en cuanto a cumplimiento. Cabe señalar, que resulta conveniente segmentar los objetivos en sub-objetivos, con el fin de favorecer y simplificar las modificaciones que se precisen realizar en las distintas áreas de la organización y lograr que el personal sea parte activa en la consecución de los resultados.

2. Determinar los indicadores: para evaluar y tener la certeza que los objetivos planteados se están consiguiendo; siendo necesario fijar estándares de desempeño aceptables que sirvan de guía para efectuar una confrontación y determinar si se debe aplicar medidas correctivas. Dichos indicadores deberán poseer características básicas, entre ellas: revelar las operaciones estratégicas para cumplir los objetivos de la organización, ser fáciles de asimilar por todos los integrantes de la empresa, evitar la subjetividad, ser sencillos de cuantificar, enfocarse a corto plazo pero considerando que la entidad pueda crecer y sea sostenible en el largo plazo.

3. Monitoreo, reporte de resultados y retroalimentación: se debe identificar dos actividades importantes: por un lado reconocer y recompensar a las áreas que cumplieron a cabalidad con los indicadores predeterminados, y por otra parte, reforzar las áreas que experimentaron dificultades para el cumplimiento de las metas planteadas e implementar gestiones para rectificar cualquier irregularidad (Ramírez, 2013). 


\section{Metodología.}

El trabajo de investigación fue no experimental, en vista que se recolectó la información de manera directa en la asociación APECAP dedicada a la producción y comercialización de café en grano y procesado, sin efectuar ningún tipo de manipulación de las operaciones, para ser examinadas posteriormente. El enfoque fue de carácter mixto por cuanto empleó herramientas cualitativas para elaborar las bases teóricas que sustentan la investigación, así mismo, se utilizó factores cuantitativos para valorar los criterios proporcionados por el personal de la asociación, permitiendo identificar patrones de comportamiento con fundamento en los resultados obtenidos. En lo referente al alcance, se empleó el método descriptivo en la interpretación de resultados del diagnóstico organizacional efectuado; el método explicativo aportó para el conocimiento detallado de los componentes del modelo de gestión integral de activos fijos. La investigación tuvo una finalidad transversal, debido a que la información se recolectó mediante visitas in situ a la asociación para conocer y poseer elementos de juicio suficientes que permitan diagnosticar la situación empresarial real, en un solo momento en el tiempo.

Los métodos empleados en la investigación fueron: el histórico - lógico, utilizado en la indagación de las diversas conceptualizaciones sobre gestión integral y control contable administrativo de activos fijos, siguiendo un orden cronológico, y efectuando un análisis crítico de los elementos que se ha ido incorporando para su complementación y perfeccionamiento; el inductivo - deductivo puesto que se partió del estudio de temáticas generales hasta centrarse en argumentos que respaldan y se configuran como la esencia de la gestión integral de los bienes; analítico - sintético que contribuyó en la desagregación de las variables, ayudando a un mejor discernimiento de sus componentes de manera individual y con posterioridad al análisis global; métodos estadísticos, para la tabulación de las encuestas, obteniendo cuadros de frecuencias y porcentajes que fueron representados gráficamente e interpretados para facilitar su comprensión; modelación, para el estructuramiento del modelo de gestión integral que se conformó por cuatro componentes principales: planificar, ejecutar, controlar y actuar.

De la misma manera, fueron útiles las siguientes técnicas e instrumentos para la investigación: encuesta, se diseñó y aplicó de manera estratificada, diferenciando en personal del departamento contable y departamentos administrativos, apoyándonos para ello en el planteamiento de preguntas estratégicas reunidas en un cuestionario; entrevista, se mantuvo una conversación con el presidente de la asociación para contrastar los datos con las respuestas de los demás colabores respecto a determinados temas de interés, para ello se diseñó una guía de preguntas; revisión documental, contribuyó a verificar y despejar dudas originadas por las inconsistencias presentadas, empleando para ello una ficha documental. 
En la presente investigación se trabajó con dos universos de estudio: el primer universo de estudio estuvo conformado por las personas que intervienen en la adquisición, manejo y registro de los activos fijos de la asociación y se muestra en la tabla 2:

Tabla 2 Población Asociación APECAP

\begin{tabular}{cccc}
\hline & Área & Cargo & No Personas \\
\hline \multirow{3}{*}{ Asociación } & Contabilidad & Contadora & 1 \\
APECAP & & Auxiliar contable & 1 \\
& Administración & Presidente & 1 \\
& Bodega & Administrador & 1 \\
& & Bodeguero & 1 \\
& & Total & $\mathbf{5}$ \\
\hline
\end{tabular}

Fuente: Asociación APECAP.

El segundo universo de estudio estuvo compuesto por las transacciones relacionadas con los activos fijos, que se ejecutan anualmente, mismas que se muestran en la tabla 3 :

Tabla 3 Transacciones anuales de activos fijos de APECAP

\begin{tabular}{lcc}
\hline \multicolumn{1}{c}{ Proceso } & $\mathbf{N}^{\circ}$ de casos & Casos a analizar \\
\hline Depreciación & 12 & 12 \\
Revalorización & 1 & 1 \\
& Total & $\mathbf{1 3}$ \\
\hline
\end{tabular}

Fuente: Asociación APECAP.

\section{Resultados.}

Los resultados de la aplicación de los instrumentos de investigación para el análisis del control contable administrativo de activos fijos en APECAP, se muestran a continuación:

\section{$\checkmark \quad$ Inventario de activos fijos}

Los activos fijos constituyen los bienes tangibles que requiere la asociación para la producción y comercialización de café molido, en tal razón, el inventario de los activos fijos es relevante en el sentido que aporta valor fundamental para el control de existencias, cumplimiento de la normativa contable y cumplimiento de la normativa tributaria.

Del diagnóstico efectuado sobre este indicador se estableció que la asociación carece de una planificación de activos fijos para cada periodo contable que le permita identificar la cantidad de recursos tangibles (bienes) que se requieren para el giro del negocio, a pesar de existir un presupuesto general, no se dispone de un presupuesto detallado de activos que se requieran 
comprar a futuro, así como no se cuenta con información específica sobre la presupuestación de venta y baja de activos fijos.

En lo referente al control de los activos fijos se evidencia la ausencia de una política de control que permita validar periódicamente las existencias, estado actual de los activos y su ubicación, además se observa una falta de consenso de los responsables del manejo contable de la empresa y del bodeguero ya que no disponen de documentos que certifiquen el levantamiento y control de los activos fijos durante los últimos doce meses.

\section{$\checkmark$ Adquisición de activos fijos}

Las actividades y acciones inherentes a la adquisición de activos fijos deben asegurar que su desarrollo se efectúe en cumplimiento de los objetivos de gestión de la entidad, por consiguiente, la identificación de partidas en el presupuesto asegurará su adecuado desarrollo. La adquisición de los activos fijos en la asociación es un proceso que responde al análisis previo de sus necesidades y a la solicitud de proformas por parte de los oferentes a fin de seleccionar los bienes que se consideran más adecuados. Para los efectos tributarios y contables estas compras se encuentran respaldadas con los comprobantes de venta e ingresados a bodega previa la revisión del cumplimiento de las características solicitadas.

Entre los criterios más relevantes identificados por los responsables del departamento contable al momento de adquirir los activos fijos prevalecen en primera instancia la calidad, luego el precio y en menor importancia la forma de pago. En cuanto al procesamiento de la información contable cabe mencionar que la totalidad de transacciones que involucran activos fijos se registraron de manera oportuna, por el importe correcto, en las cuentas correspondientes y dentro del periodo contable en que tuvieron lugar.

\section{$\checkmark$ Reparación y mantenimiento de activos fijos}

Para que un activo fijo pueda rendir y aportar con su capacidad máxima a la generación de beneficios económicos futuros, es imprescindible que se encuentre en perfectas condiciones, siendo necesario para ello recurrir a mantenimiento de tipo correctivo e incluso preventivo para mantener o de ser el caso recuperar sus características normales para el funcionamiento.

En este sentido, se determinó que la asociación APECAP planifica en forma trimestral el mantenimiento de activos fijos, sin embargo, no se da cabal cumplimiento a esta planificación, en vista que el mantenimiento se realiza cuando se percibe ligeras fallas en los activos fijos específicamente en la maquinaria y equipo.

En lo que respecta a las acciones que tienen lugar cuando un activo fijo presenta algún desperfecto, se pudo identificar que una vez que el funcionario responsable informa a la autoridad competente dicha novedad, la reparación del bien ocurre generalmente dentro de 
una semana, situación que inevitablemente genera retrasos y malestar en el desempeño de las operaciones interrumpiendo su flujo normal. En este sentido, se atribuye dicho evento a la inexistencia de una planificación de mantenimiento preventivo que evite la presencia de fallas y por tanto reducir el tiempo muerto empleado en la reparación del bien.

\section{$\checkmark$ Aseguramiento de activos fijos}

Con el propósito de garantizar el futuro económico, salvaguardar la estabilidad y proteger el patrimonio de una empresa es necesario la contratación de un seguro que cubra los posibles daños que podrían sufrir sus activos, producto de los múltiples riesgos que amenazan la actividad económica-financiera, tales como desastres naturales, incendios, hurto y otros.

En este aspecto, se constató que la asociación si bien ha contratado un seguro para los activos fijos, éste cubre únicamente el vehículo y la motocicleta, generando que los demás activos fijos se encuentran desprotegidos y expuestos a una serie de eventualidades que pueden afectar sus características físicas y funcionales, entre ellos la maquinaria y equipo que constituye su principal herramienta de trabajo para el procesamiento del café en grano.

\section{$\checkmark$ Depreciación de activos fijos}

La constante utilización de los activos fijos tanto en actividades administrativas como productivas ocasiona su desgaste físico o pérdida de valor; consecuentemente, es imprescindible emplear un mecanismo denominado depreciación, que permite asignar la disminución progresiva del valor del bien, a los costos o gastos del periodo contable, en función del área en la cual dichos bienes prestaron sus servicios.

En virtud de ello, la asociación APECAP efectúa el cálculo de la depreciación de sus activos fijos empleando el método de línea recta, sin embargo, por medio de la revisión documental se pudo corroborar la falta de control de la vida útil y depreciación de los activos fijos, inobservando las disposiciones del reglamento a la LORTI en lo que respecta al porcentaje de depreciación y años de vida útil establecidos para tal efecto, puesto que, el edificio se está depreciando para un número de años mayor al establecido por la normativa tributaria, así como también el equipo de cómputo adquirido en el año 2013 se continúa depreciando a pesar de haber concluido su vida útil; particularidades que generan gastos no deducibles que influyen en la preparación de estados financieros.

De igual manera se evidenció que no se ha diferenciado y asignado correctamente la depreciación de los activos fijos en función del área en la cual se utilizan, cargando el valor total de la depreciación a los gastos del periodo, inclusive el importe por el desgaste de la maquinaria y equipo tales como: fumigadora, despulpadora, tostadora y piladora que contribuyen directamente en la elaboración del producto, desconociendo su aporte en el proceso productivo. 


\section{$\checkmark$ Revalorizaciones}

En la actualidad, debido a las fluctuaciones innatas del mercado económico-financiero puede ocurrir que ciertos activos fijos experimenten un incremento representativo en su valor, siendo preciso ajustarlo de tal forma que se actualice el importe del patrimonio real con que cuenta la organización para la ejecución de sus operaciones.

En tal sentido, se determinó que la asociación APECAP no ha efectuado la revalorización de activos fijos tales como: edificio, terrenos y maquinaria, desconociendo el valor real de los mismos, y ocasionando por tanto que el patrimonio no refleje fidedignamente la situación de la entidad.

\section{$\checkmark$ Baja de activos fijos}

Cuando un activo fijo ha sufrido algún deterioro o daño que imposibilite su funcionamiento normal, concluido su vida útil, ha sido objeto de hurto o eventualmente ha quedado rezagado debido a los avances tecnológicos, y en este contexto, carece de capacidad para generar beneficios futuros a la entidad, se procede a desvincularlo del grupo de propiedad, planta y equipo, depurando así los bienes útiles para la empresa.

En este aspecto, en la asociación APECAP se determinó que en el último año, la Junta de Vigilancia no ha realizado una verificación y análisis de las características actuales de los activos fijos para determinar aquellos que reúnen las condiciones para ser desvinculados y dados de baja de los registros contables, en vista que no aportan al desarrollo de las operaciones organizacionales y generación de valor. No se ha realizado además un control de bienes para determinar si existen faltantes, con el fin de efectuar una investigación exhaustiva para esclarecer los hechos y determinar responsabilidades.

\section{$\checkmark$ Base de datos}

Disponer información detallada y organizada de forma correcta del grupo de activos fijos, permitirá diseñar posibles estrategias que puede implementarse para una ubicación táctica, buen uso y custodia, orientado a lograr un mayor aprovechamiento de bienes.

En virtud de ello, la asociación APECAP cuenta con registros auxiliares independientes por cada bien, mismos que se concilian con los mayores en forma trimestral, sin embargo, la última vez que se cotejó esta información fue en el año 2018; así mismo, se determinó que la base de datos que mantiene la asociación carece de información íntegra y no se encuentra actualizada, siendo necesario incluir aspectos relevantes para la buena administración, tales como: código de identificación, modelo, marca, departamento usuario, así como su estado actual. En el mismo sentido, se evidenció que la asociación carece de una técnica de identificación de activos fijos, que le permita llevar un mejor control sobre los bienes. 


\section{$\checkmark$ Determinación de procesos y responsabilidad de activos fijos}

Una entidad que pretenda una gestión eficiente deberá identificar, estructurar y documentar sus procesos en forma dinámica, estableciendo los pasos a seguir y el personal involucrado; lo cual permitirá establecer patrones de desempeño ineficaces o incluso estrategias de mejora, evitar duplicidad del esfuerzo para una misma tarea y aprovechar al máximo los recursos empresariales. Además, resulta imprescindible que el personal que utiliza los activos fijos, conozca y asuma sus responsabilidades, logrando la ejecución de sus acciones con un alto grado de compromiso, procurando el buen empleo y protección de los bienes.

Es así que, se evidenció que la asociación no cuenta con procesos claramente estructurados que guíen el desarrollo de las operaciones, desconociendo el protocolo a seguir para su ejecución, en cuanto a la segregación de funciones se identificó que el personal que interviene en la custodia de los bienes es completamente independiente a los encargados del manejo contable, sin embargo, los mismos efectúan un cruce de información para conciliar los activos físicos existentes con lo contabilizado, además se identificó que existe un jerárquico superior encargado de autorizar el uso y demás actividades que involucran activos fijos.

De igual manera, se determinó que no ha se diseñado un documento que certifique la entrega de bienes que se encontrarán bajo la custodia de los funcionarios, careciendo de un respaldo para tomar las acciones respectivas en función del tipo de responsabilidad que tuviere lugar, en caso de suscitarse alguna contingencia. En este contexto, también es vital, el aporte del bodeguero para la buena administración de los activos, sin embargo, a pesar de afirmar que se lleva un control para facilitar su identificación y ubicación, por medio de la revisión documental se determinó que no existe un registro escrito actualizado de dicha tarea.

Del mismo modo, se evidencia falta de formalidad en la socialización de las responsabilidades y prohibiciones que debe acatar el personal, pues si bien las medidas de seguridad de activos fijos se conocen a breves rasgos por sentido común, más no se ha organizado una charla y/o capacitación respecto del uso correcto y aprovechamiento de éstos.

\section{$\checkmark$ Evaluación de Resultados}

Examinar el uso correcto, rendimiento y aprovechamiento de los activos fijos de la organización a través de estándares o indicadores, proporcionará indicios de la eficiencia operativa para definir estrategias y políticas que perfeccionen los procesos y por ende el desempeño organizacional.

Es así que, se determinó que la asociación no ha efectuado un análisis del rendimiento de los activos en función del capital desembolsado para su adquisición, desconociendo si se está retribuyendo razonablemente y recuperando esta inversión; de igual manera, a pesar que el personal responsable manifiesta efectuar un estudio crítico del aprovechamiento de la 
capacidad instalada no existen registros escritos que validen la evaluación correspondiente para determinar si se están explotando al $100 \%$ de su capacidad o se están subutilizando.

\section{Propuesta.}

El modelo de gestión integral de activos fijos propuesto se efectuó con enfoque en cuatro aspectos claramente definidos: planificar, ejecutar, controlar y actuar, con el fin de mejorar la administración de la propiedad, planta y equipo que dispone la empresa (figura 2).

Figura 2. Esquema de la Propuesta de Gestión de Activos Fijos

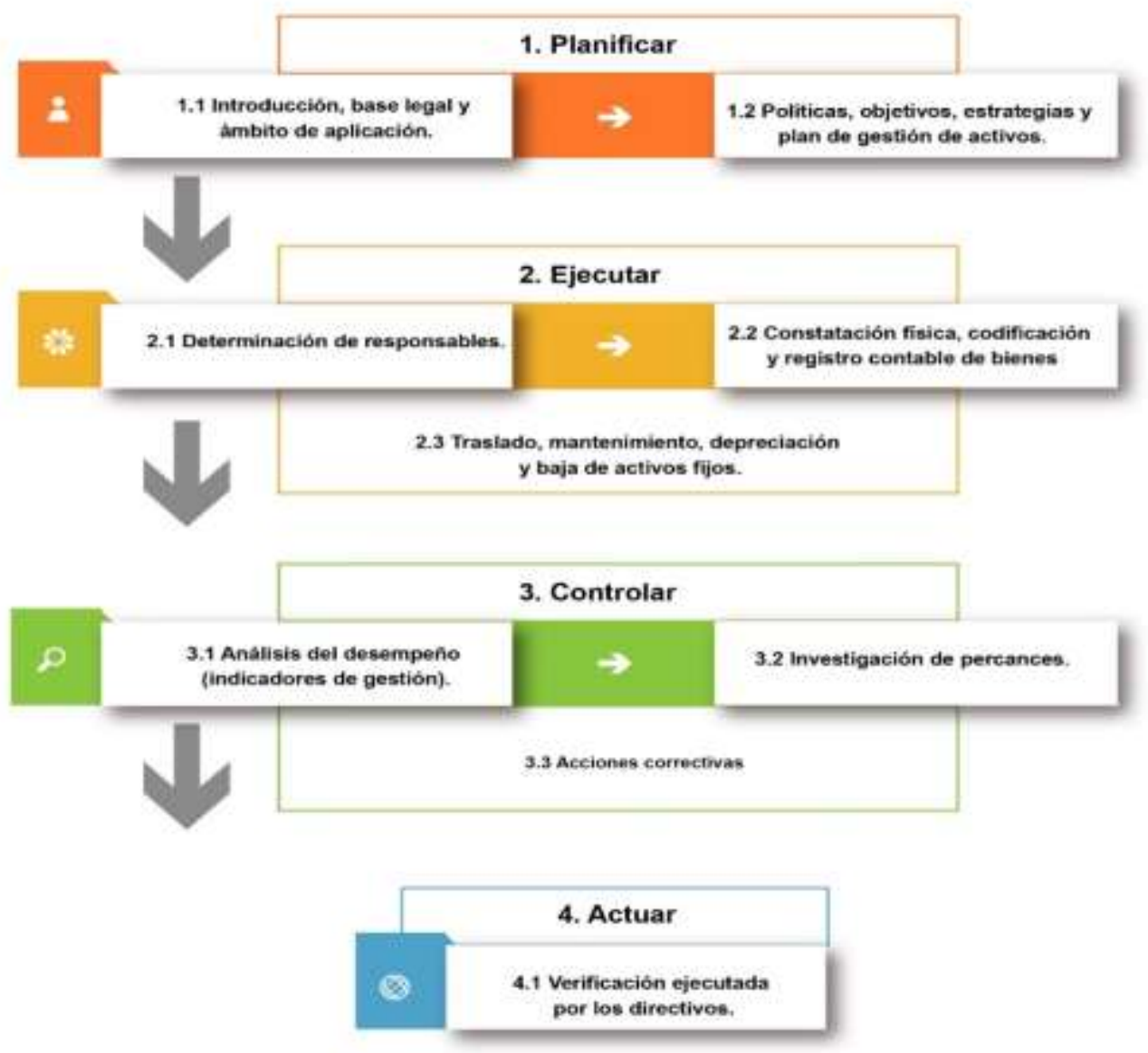

A continuación, se describe sus principales componentes:

1. Planificar: se partió de una introducción resaltando la importancia de una adecuada gestión integral de activos fijos, así mismo, el conocimiento del contexto organizacional, misión, visión, identificación de los stakeholders de la asociación, el organigrama, base legal en la cual se encuentran enmarcadas las actividades, y, especificación de los funcionarios que estarán obligados a regirse y acatar las directrices del modelo de gestión. De igual manera se estableció las principales políticas contables relacionadas con el alcance y periodicidad del conteo físico de 
activos fijos, proceso y responsabilidad para la constatación física, técnica de identificación y parámetros para el reconocimiento de un activo fijo como tal, control y aseguramiento, mejoras y mantenimiento, depreciación y baja de los activos fijos. En el mismo sentido se establecieron los objetivos del modelo propuesto, se planteó estrategias para la adecuada administración de los bienes, detallando las actividades, recursos, cronograma y responsabilidades en el plan de gestión de activos.

2. Ejecutar: tiene como punto de partida la determinación de responsabilidades, para lo cual se planteó el uso de la matriz RACI (Responsible, Accountable, Consulted, Informed), se efectuó también el estructuramiento de matrices donde se detallan los pasos a seguir y diseño de flujogramas que permiten una mejor comprensión de los procesos referentes a la constatación física, adquisición, recepción de activos fijos adquiridos, solicitud y traslado, mantenimiento y reparación de bienes, depreciación, bajas de activos fijos por venta y por chatarrización; además se abordó la técnica de codificación de los activos fijos.

3. Controlar: para evaluar y medir el nivel de desempeño de los funcionarios en los distintos procesos se propone el uso de indicadores de gestión o desempeño, los cuales permitirán identificar falencias, procesos ineficientes y demás aspectos débiles que deben ser mejorados; llevar a cabo la investigación de percances tales como hurto, pérdida, daño y otros hechos que involucren los activos fijos, para estar en capacidad de tomar las respectivas acciones correctivas.

4. Actuar: consiste en una fase de seguimiento y verificación del desarrollo, rendimiento y resultados de la implantación del modelo de gestión integral de activos fijos, que será ejecutada por los directivos de la entidad para valorar en qué medida se logró mejorar el control contable - administrativo de los activos fijos.

\section{Conclusiones.}

$\checkmark$ La gestión de activos constituye una herramienta imprescindible para aprovechar al máximo su capacidad instalada en pro del desarrollo empresarial, de igual manera es importante reconocer su desgaste, así como también cuando ha concluido su vida útil para depurar los recursos productivos con los que realmente cuenta la entidad.

$\checkmark$ El control contable y administrativo cumplen una importante función, puesto que la clave del éxito empresarial radica no solamente en la cantidad de bienes y recursos que se disponga, sino sobre todo del uso, control y protección que se ejerza sobre ellos para lograr su máximo rendimiento, así como del adecuado registro y procesamiento de la información contable.

$\checkmark$ La propuesta del modelo de gestión integral de activos fijos constituye un instrumento eficaz para que la asociación esté en capacidad de controlar eficientemente la vida útil y depreciación de los activos fijos que posee; administrar su buen uso por medio de la asignación de responsabilidades para su protección y custodia, y seguimiento de 
los procesos estructurados para su correcta ejecución, favoreciendo el cumplimiento de los parámetros de desarrollo y rentabilidad planificados.

\section{Referencias bibliográficas.}

Agudelo Pulido, A. I. (2013). Propuesta de modelo de gestión de activos fijos con enfoque en procesos. 5(2), 45-56. Recuperado el 08 de Mayo de 2018, de http://revistas.usta.edu.co/index.php/signos/article/viewFile/2181/2289

Amendola , L. (2011). Gestión integral del mantenimiento de activos como estrategia de negocios (assessment, PAS 55 - ISO 55000). 1. Valencia, España. Recuperado el 5 de Febrero de 2018, de http://www.mantenimientomundial.com/sites/mm/notas/Amendola2011.pdf

Amendola, L. (febrero de 2011). La madurez como factor de éxito en la gestión integral de activos físicos asset management PAS 55. Global Asset Management. Recuperado el 5 de Febrero de 2018, de http://www.mantenimientomundial.com/sites/mmnew/bib/notas/Factor-ExitoPAS55.pdf

Bedoya, C. (Septiembre de 2014). ISO 55000 Gestión de activos, una mirada hacia el futuro desde el área de mantenimiento. XXXI Congreso técnico FICEM, 16. Santo Domingo, República Dominicana. Recuperado el 5 de Febrero de 2018, de http://ficem.org/boletines/boletines2014/BOLETIN_DE_RESULTADOS_CT_2014 _PRESENTACIONES_CT_2014/3_MANTENIMIENTO/1_CARLOS\%20MARI O\%20BEDOYA_ARGOS/GESTION_ACTIVOS_APORTE_MTTO_2014_V4.pdf

Bernal, M. (2004). Contabilidad, sistema y gerencia. Nuevo enfoque teórico - práctico para la gestión y aplicación de la contabilidad como sistema de información. Caracas, Venezuela: CEC S.A. Recuperado el 29 de Enero de 2018, de https://books.google.com.ec/books?id=8w6XMU_drbMC\&printsec=frontcover\&dq $=$ contabilidad $\& h l=e n \& s a=X \& v e d=0 a h U K E w i N-e D j 5 e f Y A h W S 7 V M K H Y-$ nAU0Q6AEILjAB\#v=onepage $\& \mathrm{q}=$ contabilidad $\& \mathrm{f}=$ false

Candelario, J. (diciembre de 2013). Diseño e implementación de un modelo de gestión integral de activos físicos para la gestión de cadena de susministro en la industria. Protocolo doctoral tesis: diseño e implementación de un modelo de gestión integral de activos físicos para la gestión de cadena de susministro en la industria, 3. Valencia, España. Recuperado el 5 de Febrero de 2018, de http://www.upv.es/contenidos/ENCDOC/info/U0657514.pdf 
Carmenate Ávila, Y. (s.f.). Control interno en las entidades cubanas. Recuperado el 02 de mayo de 2018, de http://www.eumed.net/libros-gratis/2013/1245/importanciacontrol-interno.html

Cerón, A., Orduña, I., Aponte , G., \& Romero, A. (junio de 2015). Panorama de la gestión de activos para transformadores de potencia. La Serena, 26(3). Recuperado el 5 de Febrero de 2018, de https://scielo.conicyt.cl/scielo.php?pid=S071807642015000300014\&script=sci_arttext\&tlng=en

Contaduría General de la Nación. (2015). Procedimiento de control interno contable y de reporte del informe anual de evaluación a la Contaduría general de la nación. Recuperado el 02 de mayo de 2018, de http://www.contaduria.gov.co/wps/wcm/connect/48a87b4c-dfa2-48a1-aca67b1b53e2c1f3/Proyecto+Procedimiento+Control+Interno+Contable+(17-122015)+(1).pdf?MOD=AJPERES\&CONVERT_TO=url\&CACHEID $=48 \mathrm{a} 87 \mathrm{~b} 4 \mathrm{c}-$ dfa2-48a1-aca6-7b1b53e2c1f3

Coral, L. (2013). Contabilidad universitaria (Séptima ed.). Bogotá, Colombia: McGraw-Hill. Recuperado el 29 de Enero de 2018

Espejo, L. (2007). Contabilidad General (Primera ed.). Loja, Ecuador: Universidad Técnica Particular de Loja. Recuperado el 22 de Enero de 2018

Estupiñán Gaitán, R. (2015). Control interno y fraudes: análisis de informe COSO I, II y III con base en los ciclos transaccionales (Tercera ed.). Bogotá, Colombia: ECOE Ediciones. Recuperado el 08 de Marzo de 2018

International Accounting Standards Board. (01 de Enero de 2005). NIC 16. Recuperado el 29 de Enero de 2018, de https://www.mef.gob.pe/contenidos/conta_publ/con_nor_co/vigentes/nic/16_NIC.p df

Monge, P. (Enero - junio de 2005). Las normas internacionales de contabilidad. Actualidad contable FACES, 8(10), 36-38. Recuperado el 29 de Enero de 2018, de http://www.redalyc.org/html/257/25701005/

Norka , V. (julio - diciembre de 2005). Factores que inciden en el sistema de control interno de una organización. Actualidad contable FACES, 8(11), 87-92. Recuperado el 08 de Marzo de 2018, de http://www.redalyc.org/articulo.oa?id=25701111

Parkin, M., Esquivel, G., \& Muñoz , M. (2007). Macroeconomía (Séptima ed.). Naucalpan de Juárez, México: Pearson Educación. Recuperado el 30 de Enero de 2018, de https://books.google.com.ec/books?id=NCMmkjTxKa4C\&pg=PA115\&dq=qu\%C3 
ISSN: 2602-8506

Vol. 3, N², p. 305-328, abril - junio, 2019

$\%$ A9+es+la+depreciaci\%C3\%B3n\&hl=en\&sa=X\&ved=0ahUKEwjvudLH7pvZAh Wto1kKHUkjBsoQ6AEIJzAA\#v=onepage $\& \mathrm{q}=$ depreciaci\%C3\%B3n\&f=false

Ramírez Padilla, D. (2013). Contabilidad administrativa un enfoque estratégico para competir (Novena ed.). México: Mc Graw Hill .

Santos Jiménez, N. (s.f.). Decisión de comprar o alquilar activos fijos. Revista de la Facultad de Ingeniería Industrial, 11, 9-17. Recuperado el 02 de mayo de 2018, de https://www.redalyc.org/html/816/81619829002/

Schermerhorn, J. (2010). Administración (Segunda ed.). (G. Noriega, Ed.) México: Limusa Wiley. Recuperado el 12 de marzo de 2018 


\section{PARA CITAR EL ARTÍCULO INDEXADO.}

García L., Narváez C. \& Erazo J., (2019). Gestión integral de activos fijos con enfoque en el control contable administrativo., revista electrónica Visionario Digital 3(2), 305-328. Recuperado desde: http://www.visionariodigital.org

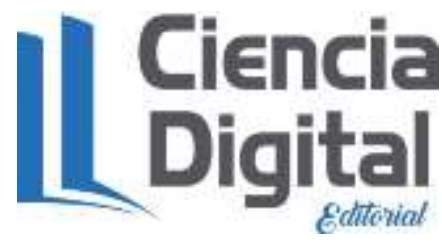

El artículo que se publica es de exclusiva responsabilidad de los autores y no necesariamente reflejan el pensamiento de la Revista Ciencia Digital.

El artículo queda en propiedad de la revista y, por tanto, su publicación parcial y/o total en otro medio tiene que ser autorizado por el director de la Revista Ciencia Digital.
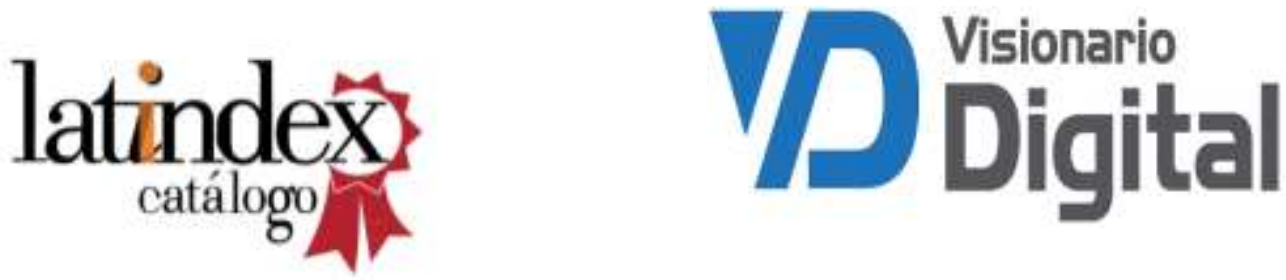